\title{
DEGENERATE CONICS REVISITED
}

Dedicated to Armin Herzer on his 60th birthday.

Hans Havlicek

In a desarguesian projective plane we discuss those degenerate conics whose parameters have degree two over the centre of the underlying skew field. Such degenerate conics are closely related with Baer subplanes. This relationship enables us to improve and reformulate in a geometric language some theorems which previously have been established in a purely algebraic way. A geometric description of the family of fundamental chains of these degenerate conics is given.

1. Throughout this article $K$ denotes a non-commutative field. $Z(L)$ stands for the centralizer in $K$ of $L \subset K$. If $L \subset K$ is a (skew or commutative) subfield, then a right vector space $\mathfrak{B}$ over $L$ is also written as $\mathfrak{B}_{L}$. We shall frequently regard $K$ as a right vector space $K_{L}$ over $L$. The affine and projective space on $\mathfrak{B}_{L}$ is denoted by $\mathscr{A}\left(\mathfrak{B}_{L}\right)$ and $\mathcal{P}\left(\mathfrak{B}_{L}\right)$, respectively. Let $(\mathscr{Y}, \mathscr{L})$ be a linear space with point set $\mathscr{I}$ and line set $\mathscr{L}$. Given $\mathscr{T} \subset \mathscr{I}$ then put $\mathscr{L}_{\mathscr{T}}:=\{\ln \mathcal{T}|l \in \mathscr{L} \wedge| \ln \mathcal{T} \mid \geq 2\}$. Hence $\left(\mathcal{T}, \mathscr{L}_{\mathscr{T}}\right)$ is the trace space of $(\mathscr{Y}, \mathscr{L})$ determined by $\mathscr{T}$.

The reader is referred to $[10,62-73],[16]$ and $[18,325-333]$ for definitions and results on degenerate conics. Our exposition follows [10].

Suppose that $\mathcal{P}\left(\mathfrak{B}_{K}\right)$ is a projective plane on a vector space $\mathfrak{B}_{K}$ and write $\mathscr{L}$ for the set of lines of $\mathcal{P}\left(\mathfrak{B}_{K}\right)$. Let $\Gamma$ be a degenerate conic of $\mathcal{P}\left(\mathfrak{B}_{K}\right)$. Then there exists an ordered basis $(\mathfrak{p}, \mathfrak{q}, \mathfrak{a})$ of $\mathfrak{B}_{K}$ and an element $a \in K \backslash Z(K)$ such that the proper and improper part of (1) $\Gamma=\Gamma^{0} \cup \Gamma^{\times}$

are given by

(2) $\Gamma^{\circ}=\{(p t+q t a+a) K \mid t \in K\}$

and

(3) $\Gamma^{\times}=P Q$ with $P=\mathfrak{p} K, Q=q K$,

respectively $[10,65]$. In the terminology of $[18] \Gamma^{\circ}$ is a $C$-configuration. The element $a$ is called a parameter of $\Gamma$. Neither $(\mathfrak{p}, \mathfrak{q}, \mathfrak{a})$ nor $a$ is uniquely determined. We denote by $A$ the subfield 
of $K$ which is generated by $Z(K) \cup\{a\}$. Any two parameters of $\Gamma$ are in $A$ and have the same degree ${ }^{1}$ over $Z(K)[10,67]$. The set

(4) $n=\{(p t+q t a) K \mid t \in K\}$

is formed by all points on $P Q$ which are collinear with at least two different points of $\Gamma^{\circ}[10,65]$.

THEOREM 1. Let $\Gamma^{\circ}$ be the proper part of a degenerate conic $\Gamma$ with parameter $a \in K \backslash Z(K)$. Then the trace space $\left(\Gamma^{\circ}, \mathscr{L} \Gamma^{\circ}\right)$ is isomorphic to the affine space $A\left(K_{Z(A)}\right)$ and $\left(\Gamma^{\circ}, \mathscr{L} \Gamma^{\circ}\right)$ is an affine plane if, and only if, the degree $|a: Z(K)|=2$.

Proof. The subset $\mathfrak{B}:=\{p t+q t a \mid t \in K\}$ of $\mathfrak{B}$ is closed under linear combinations with coefficients in $Z(A)$, i.e. $B$ is a right vector space over $Z(A)$. The map

$$
\iota: K \rightarrow \mathfrak{B}, t \mapsto p t+q t a,
$$

is an isomorphism of the vector spaces $K_{Z(A)}$ and $\mathfrak{B}_{Z(A)}$. By (2), there exists a bijection

$$
\varepsilon: \mathfrak{B} \rightarrow \Gamma^{0}, \mathfrak{p} t+q t a \mapsto(p t+q t a+\mathfrak{a}) K .
$$

If points $t_{0}, t_{1}, t_{2}$ are collinear in $\mathscr{A}\left(K_{\mathrm{Z}(A)}\right)$, then their images under $\iota$ and $\iota \varepsilon$ are collinear in $\mathscr{A}\left(\mathfrak{B}_{Z}(A)\right.$ ) and $\mathcal{P}\left(\mathfrak{B}_{K}\right)$, respectively. On the other hand, given three pairwise different collinear points $\left(p t_{i}+q t_{i} a+a\right) K \quad(i=0,1,2)$ in $\mathcal{P}\left(\mathfrak{B}_{K}\right)$ there are uniquely determined elements $r, s \in K$ with $r+s=1$ and $t_{2}=t_{0} r+t_{1} s$. By collinearity, $t_{2} a=t_{0} a r+t_{1} a s$, whence $r, s \in Z(A)$. This in turn shows that $\mathscr{A}\left(K_{Z(A)}\right)$ and $\left(\Gamma^{\circ}, \mathscr{L} \Gamma^{\circ}\right)$ are ismorphic linear spaces.

The dimension of $\left(\Gamma^{\circ}, \mathscr{L} \Gamma^{\circ}\right)$ equals the right degree $|K: Z(A)|$. By the centralizer theorem (cf. e.g. $[9,42]$ ), $|K: Z(A)|=2$ if, and only if, $|A: Z(K)|=|a: Z(K)|=2$ as required. $\square$

Since $|Z(A)| \geq 3$, there is a unique parallelism in $\left(\Gamma^{\circ}, \mathscr{L} \Gamma^{\circ}\right)$ which coincides with the image under $\iota \varepsilon$ of the parallelism relation on $\mathscr{A}\left(K_{Z(A)}\right)$. Two lines of $\left(\Gamma^{\circ}, \mathscr{L} \Gamma^{\circ}\right)$ are parallel if, and only if, their hulls in $\mathcal{P}\left(\mathfrak{B}_{K}\right)$ have a common point on $n$. Thus the points of $n \subset P Q$ may be identified with the hyperplane at infinity ${ }^{2}$ of the affine space $\left(\Gamma^{0}, \mathscr{L} \Gamma^{\circ}\right)$. If $|K: Z(A)|=2$, then $\left(\Gamma^{\circ}, \mathscr{L} \Gamma^{\circ}\right)$ is an affine plane and $n$ is a $Z(A)$-chain of $P Q$. As an immediate consequence of Theorem 1 and $|K: Z(A)|=|a: Z(K)|=2$ we infer the following

\footnotetext{
${ }^{1}$ All degrees written down in this articles are right degrees. Cf. however Theorem 3.1.2 in [8,31].

${ }^{2}$ This is one of those projective spaces discussed in [11].
} 
COROLLARY. If $|a: Z(K)|=2$, then $\left(\Gamma^{\circ}\right.$ un, $\mathscr{L}^{\circ}$ un $)$ is a Baer subplane of $\mathcal{P}\left(\mathfrak{B}_{K}\right)$ with $\mathrm{Z}(A)$ as underlying field.

2. The following sections of this article are subject to the assumption

$$
|a: Z(K)|=2 \text {, }
$$

whence there exist $m_{0}, m_{1} \in Z(K)$ such that

(5) $a^{2}=m_{0}+m_{1} a$.

We shall frequently make use of the identity $a^{-1} m_{0}=\left(a-m_{1}\right)$ without further notice.

The group $\mathbf{G}$ of automorphic collineations of $\Gamma$ has been determined in $[10,71]$. Now we have an alternative description of $\mathbf{G}$ :

If $\gamma \in \mathrm{G}$, then $\gamma \mid \Gamma^{\circ}$ is an affinity of $\left(\Gamma^{\circ}, \mathscr{L} \Gamma^{\circ}\right)$. Conversely, let $\beta$ be an affinity of $\left(\Gamma^{0}, \mathscr{L} \Gamma^{\circ}\right)$. Hence $\varepsilon \beta \varepsilon^{-1}$ is product of a translation and a semi-linear isomorphism of $\mathbb{B}_{Z}(A)$ with respect to $\dot{b} \in \operatorname{Aut}(Z(A))$, say. This $\beta$ is extendable to a collineation $\gamma$ of $\mathcal{P}\left(\mathfrak{B}_{K}\right)$ if, and only if, $\dot{b}$ is restriction of an automorphism of $K$. If $\gamma$ exists $^{3}$, then $\gamma \in \mathbf{G}$.

3. It is our goal to discuss the partition of $P Q \backslash n$ which is given by the fundamental chains of $\Gamma[10,69]$. This will be done in terms of affine geometries which may be regarded as affine derivations of the geometry of $L$-chains on $P Q[5,320]$, where $L$ denotes a proper subfield of $K$. But first we give an alternative description of fundamental chains:

THEOREM 2. Let $\Psi$ be the group of projective collineations of $\mathcal{P}\left(\mathfrak{B}_{K}\right)$ leaving $\Gamma^{\circ}$ un pointwise invariant ${ }^{4}$. The orbit $X^{\Psi}$ of any $X \in P Q \backslash n$ equals the only fundamental chain $c_{X}$ of $\Gamma$ passing through $X$.

Proof. Suppose $\psi \in \Psi$. Clearly $X^{\psi} \in c_{X}$ for $X^{\psi}=X$. Restricting $\psi$ to the pencil of lines with vertex $X$ yields a projectivity $\alpha$, say. If $X \neq X^{\psi}$, then $\Gamma$ is a subset of the point set generated by $\alpha$ $[10,43]$, since $(P Q)^{\alpha}=P Q$ and $Y=(X Y) \cap(X Y)^{\alpha}$ for all $Y \in \Gamma^{\circ}$. But every line through $X$ other than $P Q$ intersects $\Gamma^{\circ}$, as follows from the fact that $X$ is element of a fundamental pair of $\Gamma[10,69]$.

\footnotetext{
${ }^{3}$ The collineation $\gamma$ need not exist. Take the field $H$ of real quaternions. The centralizer of any $a \in \mathbb{H} \backslash \mathbb{R}$ where $\mathbb{R}=Z(\mathbb{H})$ is a field of complex numbers $\mathbb{C}$, say. Then there exists $\dot{b} \in$ Aut $(\mathbb{C})$ such that $\mathbb{R}$ is not invariant, whence has $\dot{b}$ has no extension to $\mathbb{H}$. Cf. e.g. [14], [17].

${ }^{4}$ Any $\psi \in \Psi$ is a quasiperspective collineation.
} 
Hence $\alpha$ is a generating map of $\Gamma$ and $X^{\Psi} \subset c_{X}$.

On the other hand, assume $X=(\mathfrak{p} v+q w) K \in P Q \backslash n$. We read off from formula (3.21) in [10,69]

$$
c_{X}=\left\{\left(\mathfrak{p}\left(w-m_{1} v+q m_{0} v\right) z_{0}+(\mathfrak{p} v+q w) z_{1}\right) K \mid(0,0) \neq\left(z_{0}, z_{1}\right) \in Z(K)^{2}\right\} .
$$

Those maps of $G L\left(\mathfrak{B}_{K}\right)$ having matrices

$$
\left(\begin{array}{ccc}
z_{1}-m_{1} z_{0} & z_{0} & 0 \\
m_{0} z_{0} & z_{1} & 0 \\
0 & 0 & \left(a-m_{1}\right) z_{0}+z_{1}
\end{array}\right),(0,0) \neq\left(z_{0}, z_{1}\right) \in Z(K)^{2},
$$

with respect to the ordered basis $(\mathfrak{p}, \mathfrak{q}, \mathfrak{a})$ induce a subset $\Phi$ of $\Psi$, since every vector $\mathfrak{p} t+\mathfrak{q} t a+\mathfrak{a}(t \in K)$ is eigenvector with

$$
\left(a-m_{1}\right) z_{0}+z_{1}=m_{0} z_{0} a^{-1}+z_{1} \neq 0
$$

being its (right) eigenvalue. (In fact $\Phi=\Psi$.) By construction, $X^{\Psi} \supset X^{\Phi}=c_{X}$ which completes the proof. $\square$

We remark that a non-projective collineation $v$ which leaves $\Gamma^{\circ}$ un elementwise invariant takes $X \in P Q \backslash n$ to $X^{\nu} \notin c_{X}$ : Firstly, $X \neq X^{\nu}$ as there is no subline of $P Q$ which is properly between $n$ and $P Q$ due to $|K: Z(A)|=2$. Secondly, assume $X \neq X^{\nu} \in c_{X}$. The restriction of $v$ to the pencil of lines with vertex $X$ is a bijection $\mu$, say, such that $\Gamma^{\circ}=\left\{X Y \cap(X Y)^{\mu} \mid Y \in \Gamma^{\circ}\right\}$. But $\left(X, X^{\nu}\right)$ is a fundamental pair of $\Gamma$. Hence $\mu$ is a projectivity which generates $\Gamma$, a contradiction.

Let $\left\{b_{0}, b_{1}\right\}$ be a basis of $K_{Z(A)}$ and write $\mathfrak{b}_{i}:=\mathfrak{p} b_{i}+\mathfrak{q}_{i} b_{i}$. Then $n=\left\{\left(\mathfrak{b}_{0} t_{0}+\mathfrak{b}_{1} t_{1}\right) K \mid(0,0) \neq\left(t_{0}, t_{1}\right) \in Z(A)^{2}\right\}$. We use $\mathfrak{b}_{0}, \mathfrak{b}_{1}$ to define an affine coordinatization

$$
\sigma: P Q \backslash\left\{\mathfrak{b}_{1} K\right\} \rightarrow K, \quad\left(\mathfrak{b}_{\mathrm{O}}+\mathfrak{b}_{1} t\right) K \mapsto t
$$

We shall describe the partition of $K \backslash Z(A)=(P Q \backslash n)^{\sigma}$ given by the orbits of $\Psi^{\sigma}:=\sigma^{-1} \Psi \sigma$. The re-transfer under $\sigma^{-1}$ is immediate.

The group $\Psi^{\sigma}$ is formed by all inner automorphisms of $K$ such that $x \mapsto c x c^{-1}$ with $c \in A \backslash\{0\}$, since $Z(Z(A))=A$ by the centralizer theorem $[9,42]$. If $c=a z_{0}-z_{1}\left(z_{0}, z_{1} \in Z(K)\right)$, then

and

$$
c^{-1}=\left(\left(a-m_{1}\right) z_{0}+z_{1}\right)\left(m_{0} z_{0}^{2}+m_{1} z_{0} z_{1}-z_{1}^{2}\right)^{-1}
$$

(6) $c x c^{-1}=\left(a x a^{-1} m_{0} z_{0}^{2}+\left(a x-x a+m_{1} x\right) z_{0} z_{1}-x z_{1}^{2}\right)\left(m_{0} z_{0}^{2}+m_{1} z_{0} z_{1}-z_{1}^{2}\right)^{-1}$. $\Psi^{\sigma}$ is a subgroup of the Galois group of $K \mid Z(A)[8,40]$, but we are only concerned with inner automorphisms. (Cf. [1,283], [2], [3], [4], [5,176] for geometric interpretations of Galois groups.) However if $|K: Z(K)|<\infty$, then $\operatorname{id}_{Z(A)}$ is only extendable to inner automorphisms of $K$ (cf. e.g. the Corollary in $[8,46]$ ) and consequently every collineation which leaves $\Gamma^{\circ}$ un elementwise 
invariant belongs to $\Psi$.

See $[1,280]$ or Theorem 23 in $[15,126]$ for results similar to the following

LEMMA. In the affine plane $A\left(K_{Z(A)}\right)$ the group $\Psi^{\sigma}$ is a group of perspective affinities with common axis $Z(A)$. The pencil of lines parallel to $\left(x-a x a^{-1}\right) Z(A) \quad(x \in K \backslash Z(A)$ arbitrary) is elementwise invariant under all transformations of $\Psi^{\sigma}$. The element $a \in K$ is inseparable over the centre of $K$ if, and only if, $\Psi^{\sigma}$ is a group of shears.

Proof. Clearly $\Psi^{\sigma}$ is a group of perspective affinities with common axis $\mathrm{Z}(A)$. We deduce from

(7) $a x-x a+m_{1} x=x\left(m_{1}-a\right)+\left(a x a^{-1}\right) a$

and (6) that the orbit of $x \in K \backslash Z(A)$ under $\Psi^{\sigma}$ is a subset of the line joining $x$ and $a x a^{-1}$. Thus all lines parallel to this one are invariant under all affinities of $\Psi^{\sigma}$ and every orbit of $\Psi^{\sigma}$ is subset of such an invariant line. The group $\Psi^{\sigma}$ is a group of shears if, and only if,

$$
\left(x-a x a^{-1}\right) \in Z(A) \text { for all } x \in K
$$

or, in other words,

$$
a\left(x-a x a^{-1}\right) a^{-1}=\left(x-a x a^{-1}\right)\left(m_{1} a^{-1}-1\right)=x-a x a^{-1} \text {, }
$$

where the first sign of equality follows from a streightforward calculation. But $m_{1} a^{-1}-1=1$ is equivalent to $\operatorname{Char} K=2$ and $m_{1}=0$ which in turn characterizes a as inseparable over $\mathrm{Z}(K)$.

Suppose that $\Psi^{\sigma}$ is no group of shears. Then every $\Psi^{\sigma}$-invariant line $x+\left(x-a x a^{-1}\right) Z(A)$ where $x \in K \backslash Z(A)$ contains exactly one point $x^{\pi} \in \mathrm{Z}(A)$ which is given by

(8) $x^{\pi}=\left(a x a^{-1}\left(2 m_{0}+m_{1} a\right)+x\left(2 m_{0}-m_{1} a+m_{1}^{2}\right)\right)\left(4 m_{0}+m_{1}^{2}\right)^{-1}$.

Hence $\pi: K \rightarrow Z(A), \quad x \mapsto x^{\pi}$, is a projection in $K_{Z(A)}$. Now let $\Psi^{\sigma}$ be a group of shears. Here we put

(9) $x^{\pi}:=a x-x a=a x+x a$.

This map $\pi: K \rightarrow Z(A), x \mapsto x^{\pi}$, is $Z(A)$-linear with kernel $Z(A)$ and image $\mathrm{Z}(A)$.

In the affine space ${ }^{5} A\left(K_{A}\right)$ the orbit $c_{x}$ of $x \in K \backslash Z(A)$ under $\Psi^{\sigma}$ is a subset of a line $M_{X}$, because (6) and (7) are linear combinations with coefficients in $A$. If $a$ is separable over $Z(K)$,

${ }^{5} A=\mathrm{Z}(A)$ if, and only if, $A$ is a maximal commutative subfield of $K$, whence $|K: Z(K)|=4$ by $(5)$, formula $(10)$ in $[9,40]$ and Theorem 4 in $[9,45]$. 
then $M_{x}$ is given by $x+\left(x-x^{\pi}\right) A$. All such lines $M_{y}$ within $x+\left(x-x^{\pi}\right) Z(A)$ are incident with $x^{\pi}$. If a is inseparable over $Z(K)$, then the line $M_{x}$ equals $x+x^{\pi} A$ and all such lines $M_{y}$ within the subspace $x+x^{\pi^{x}} Z(A)$ are parallel to $x^{\pi} A$.

Now, finally, take the affine space $\mathscr{A}\left(K_{Z(K)}\right)$. Our previous discussions show that $c_{x}$ lies in the $\Psi^{\sigma}$-invariant plane $M_{x}$ which in turn is contained in the $\Psi^{\sigma}$-invariant subspace $x+\left(x^{\pi^{x}}-x\right) Z(A)$ or $x+x^{\pi} Z(A)$ in the separable or inseparable case, respectively. The configurational properties of these planes and subspaces of $\mathscr{A}\left(K_{Z(K)}\right)$ follow immediately from the corresponding results in $\mathscr{A}\left(K_{A}\right)$ and $\mathscr{A}\left(K_{\mathrm{Z}(A)}\right)$. Thus we can restrict our investigations to a fixed plane $M_{X}$.

It will be conveniant to use the projective closure

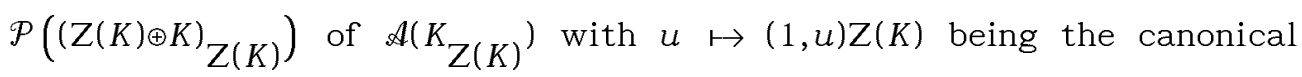
embedding. In this projective space the orbit $c_{x}$, as is given by (6), may be re-written as the set of all points

$$
\left(\left(m_{0}, a x a^{-1} m_{0}\right) z_{0}^{2}+\left(m_{1}, a x-x a+m_{1} x\right) z_{0} z_{1}+(-1,-x) z_{1}^{2}\right) Z(K)
$$

with $(0,0) \neq\left(z_{0}, z_{1}\right) \in Z(K)^{2}$. Thus $c_{x}$ is a conic without points at infinity or, in other words, an ellipse. Cf. e.g. [7,31]. This is in accordance with [5,225]. Recall that the tangents of $c_{x}$ in $\left(m_{0}, a x a^{-1} m_{0}\right) Z(K)$ and $(-1,-x) Z(K)$ intersect each other in

(10) $\left(m_{1}, a x-x a+m_{1} x\right) Z(K)$.

Let us return to affine terms: The plane $M_{x} \subset K$ carries the affine Möbius-geometry $\mathcal{A}(Z(K), A)$ in the sense of [5,101] (cf. also [12,221] and [13]) when identifying

(11) $t \in A$ with $y_{0}+\left(y_{1}-y_{0}\right) t \in M_{x}$

for any two different $y_{0}, y_{1} \in M_{x}$. This identification does not essentially depend on $y_{0}, y_{1}$, because it is unique to within circle preserving affinities of $\mathscr{A}(Z(K), A)$. Hence we may speak unambigously of circles within the plane $M_{x}$. (Letting $t \in K$ in (11) yields a bijection $K \rightarrow K$ which is a circle preserving affinity of the affine Möbius-geometry $\mathscr{A}(Z(K), K)$.)

THEOREM 3. The orbit $c_{x}$ of $x \in K \backslash Z(A)$ under $\Psi^{\sigma}$ is a circle of the plane $M_{X}$

Case 1 - a is separable over $\mathrm{Z}(K)$ : The mid-point (Char $K \neq 2$ ) or nucleus (Char $K=2$ ) of the circle $c_{x} \subset M_{x}$ is the point $x^{\pi} \in Z(A)$ as given by (8). A circle of $M_{x}$ is an orbit of $\Psi^{\sigma}$ if, and only if, its mid-point respectively nucleus equals $x^{\pi}$.

Case 2 - a is inseparable over $\mathrm{Z}(K)$ : All tangents of $c_{x}$ are 
parallel to the line $x^{\pi} \mathrm{Z}(K)$ with $x^{\pi}$ given by (9). A circle of the plane $M_{x}$ is an orbit of $\Psi^{\sigma}$ if, and only if, there exists a translation mapping it into $c_{x}$.

Proof. The orbit $c_{x}$ is a circle of $M_{x}$, since its image under $\sigma^{-1}$ is a $Z(K)$-chain of $P Q$.

Case 1: For Char $K=2$ we deduce from $m_{1} \neq 0$ and (10) that the nucleus of $c_{x}$ is not at infinity. The mid-point respectively nucleus of $c_{x}$ is invariant under all transformations of $\Psi^{\sigma}$, whence it has to be $x^{\pi}$. But $y^{\pi}=x^{\pi}$ for all $y \in M_{x}$, so that all orbits $c_{y}$ $\left(y \in M_{X} \backslash Z(A)\right)$ have common mid-point or nucleus, respectively.

Conversely, let $c$ be a circle of $M_{x}$ with mid-point respectively nucleus $x^{\pi}$. Choose $y \in c$. As a circle is uniquely determined by one of its points and its mid-point respectively nucleus, we get $c=c_{y}$.

Case 2: We read off from (10) together with CharK $=2$ and $m_{1}=0$ that all tangents of $c_{X}$ are parallel to $x^{\pi} Z(K)$.

If $y \in M_{x}$, then $y=x+x^{\pi} s$ with $s \in A$ and $c y c-1=c x c^{-1}+x^{\pi} s$. Thus the translation $\tau: M_{x} \rightarrow M_{x}, u \mapsto u+x{ }_{s}$, maps $c_{x}$ into $c_{y}$. The proof is completed by reversing the above arguments. $\square$

We remark that in case 2 there are circles of $M_{x}$ whose tangents are parallel to $x^{\pi} Z(K)$ without being orbits of $\Psi^{\sigma}$. An example is given by a circle which touches an orbit of $\Psi^{\sigma}$ in exactly one point.

Finally, take as $K$ the real quaternions $\mathbb{H}$. Then $\mathcal{M}_{x}$ is a plane of complex numbers and $\Psi^{\sigma}$ may be interpreted as a full group of rotations of the euclidian space $\mathscr{A}(\mathbb{R}, \mathbb{H})$ with a pointwise invariant plane. Inner automorphisms of $\mathbb{H}$ (cf. formula (6)) are a standard tool for the representation by quaternions of motions of the euclidian 2-sphere. Cf. e.g. [6].

\section{REFERENCES}

[1] BAER, R.: Projectivities with fixed points on every line of the plane, Bull. Americ. math. Soc. 52, 273 - 286 (1946).

[2] BENZ, W.: Die Galoisgruppen als Gruppen von Inversionen, Math. Ann. 178, 169 - 172 (1968).

[3] BENZ, W.: Nichtkommutative Möbiusgeometrie, Math. Nachr. 38, 349 - 359 (1968).

[4] BENZ, W.: Zur Geometrie der Körpererweiterungen, Canadian J. Math. 21, 1097 - 1122 (1969).

[5] BENZ, W.: Vorlesungen über Geometrie der Algebren, Grundlehren Bd. 197, Berlin - Heidelberg - New York, Springer, 1973.

[6] BLASCHKE, W.: Kinematik und Quaternionen, Berlin, VEB Dt. Verlag d. Wissenschaften, 1960.

[7] BRAUNER, H.: Geometrie projektiver Räume II, Mannheim - Wien - 
Zürich, BI - Wissenschaftsverlag, 1976.

[8] COHN, P.M.: Skew Field Constructions, London Math. Soc. Lect. Notes Ser. 27, Cambridge, Cambridge University Press, 1977.

[9] DRAXL, P.K.: Skew Fields, London Math. Soc. Lect. Notes Ser. 81, Cambridge, Cambridge University Press, 1983.

[10] HAVLICEK, H.: Applications of Results on Generalized Polynomial Identities in Desarguesian Projective Spaces. In: R. KAYA, P. PLAUMANN and K. STRAMBACH (eds.): Rings and Geometry, Dordrecht, D. Reidel, 1985.

[11] HAVLICEK, H.: Durch Kollineationsgruppen bestimmte projektive Räume, Beitr. Algebra Geom. 27, 175 - 184 (1988).

[12] HERZER, A.: Über die Darstellung affiner Ketten als Normkurven, Abh. math. Sem. Univ. Hamburg 55, 211 - 228 (1985).

[13] HERZER, A.: Synthetische Konstruktion affiner Möbiusgeometrien, Arch. Math. 46, 91 - 96 (1986).

[14] NOETHER, E.: Die Funktionalgleichungen der isomorphen Abbildung, Math. Ann. 77, 536 - 545 (1916).

[15] PICKERT, G.: Projektive Ebenen, Grundlehren Bd. 80, Berlin Heidelberg - New York, Springer, 1975.

[16] RIESINGER, R.: Entartete Steinerkegelschnitte in nichtpapposschen Desarguesebenen, Monatsh. Math. 89, 243 - 251 (1980).

[17] SEGRE, B.: Gli automorfismi del corpo complesso, ed un problema di Corrado Segre, Atti Accad. Naz. Lincei, VIII. Ser., Rend., Cl. Sci. Fis. Mat. Nat. 36, 414 - 420 (1947).

[18] SEGRE, B.: Lectures on Modern Geometry, Roma, Ed. Cremonese, 1962.

Hans Havlicek

Institut für Geometrie

Technische Universität

Wiedner Hauptstraße $8-10$

A-1040 Wien

Austria 